\title{
Using serum albumin to predict frailty in the vascular perioperative patient
}

\author{
Authors: Nathan Spence, ${ }^{A}$ David Lewis, ${ }^{B}$ Fiona Windsor ${ }^{A}$ and Lauren Richardson ${ }^{A}$
}

In recent years there has been a growing interest into measuring frailty in surgical patients, and its potential impact on morbidity and mortality. Specifically, frailty scores can be used to identify patients early in their admission to ensure prompt referral to perioperative physicians or geriatricians. Our local perioperative medicine team (led by acute, renal and geriatric physicians) have recently started to work alongside the vascular surgeons to care for emergency and elective inpatients. There is now robust evidence to demonstrate that more frail vascular patients undergoing revascularisation procedures have an increased risk of poor outcomes, namely death and needing to progress to limb shortening surgery. ${ }^{1}$ These studies also explore the concept of biomarkers of frailty. Serum albumin is of particular interest to us. As a small team, one of the initial challenges we faced was determining which patients we should review.

We performed an Edmonton Frailty Score on new admissions to the vascular ward. We have collected a test data set of 2 months' worth of patients $(n=33)$. Each patient had their age, frailty score and albumin on admission recorded. The Edmonton score for each patient was calculated by the same two doctors in collaboration. Serum albumin was measured within 24 hours of admission, and always before any surgical intervention.

There was no correlation between age and frailty. There was also no correlation between age and albumin on admission (Fig 1). When frailty was plotted against albumin, a clear negative correlation emerges (Fig 2).

We continue to collect data on all new admissions to the vascular surgery ward. We are also collecting similar data for major trauma, hip fracture and emergency general surgery patients. We aim to identify if serum albumin can be used as a surrogate marker of frailty and whether this can accurately predict which patients will benefit from comprehensive medical review or geriatric assessment, in addition to usual surgical care.

Authors: ${ }^{A}$ Oxford University Hospitals NHS Trust, Oxford, UK; ${ }^{B}$ John Radcliffe Hospital, Oxford, UK

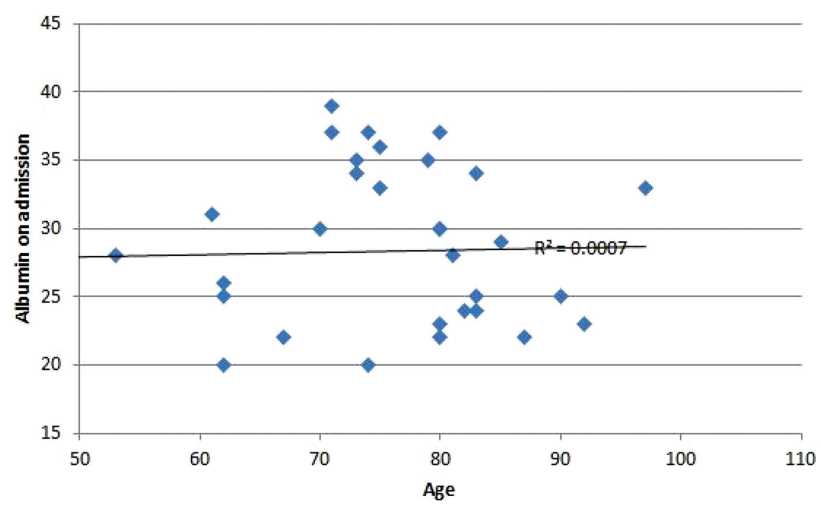

Fig 1. Patient age plotted against serum albumin on admission $(n=33)$.

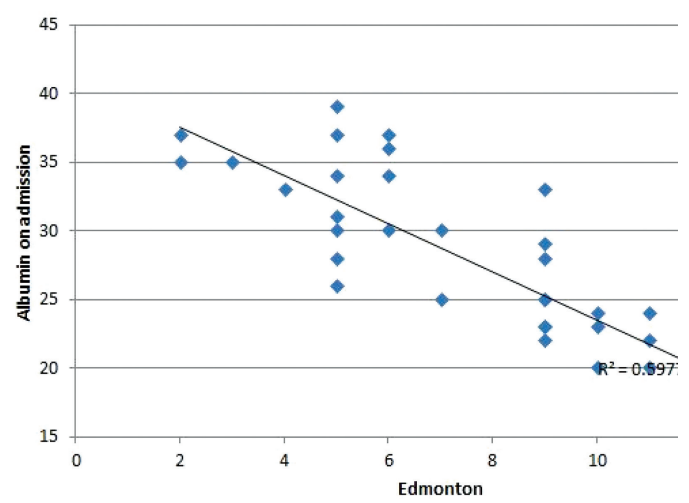

Fig 2. Edmonton Frailty Score plotted against serum albumin on admission $(n=33)$.

\section{Conflicts of interest}

None declared.

\section{Reference}

1 Donald GW, Ghaffarian AA, Isaac F et al. Frailty and biomarkers of frailty predict outcome in veterans after open and endovascular revascularization. J Surg Res 2019;243:539-52. 